\title{
Ângulo da cifose torácica em diferentes posições simulando o velejo no kitesurfing
}

http://dx.doi.org/10.11606/1807-5509201700040777

\author{
Rodrigo Fagundes ANGELLOS ${ }^{* * * *}$ \\ Lara Elena GOMES**** \\ Max William RUSCH ${ }^{* * * * *}$ \\ Verlaine Balzan LAGNI ${ }^{* * * * * * *}$ \\ Jefferson Fagundes LOSS ${ }^{* * * *}$
}

*Centro Universitário

Metodista do Instituto

Porto Alegrense,

Porto Alegre, RS

Brasil.

**Universidade

Federal de Ciências

da Saúde de Porto

Alegre, Porto Alegre,

RS, Brasil.

${ }^{* * *}$ Colegiado de

Educação Física,

Universidade Federal

do Vale do São

Francisco, Petrolina,

PE, Brasil.

${ }^{* * * *}$ Escola de

Educação Física,

Universidade Federa

do Rio Grande do

Sul, Porto Alegre, RS,

Brasil.

*****Universidade

Federal do Rio

Grande do Sul, Porto

Alegre, RS, Brasil.

\section{Resumo}

No kitesurfing, o indivíduo utiliza a energia do vento para deslizar com uma prancha na água com um kite, que é preso ao praticante com o auxilio de um dispositivo denominado trapézio. Para realizar diversos movimentos, o velejador alterara a posição do seu corpo, modificando a posição da sua coluna vertebral. Contudo, pouco se sabe sobre a postura adotada no kitesurfing, a qual pode contribuir para a ocorrência de dor nas costas. Assim, o objetivo principal foi comparar os ângulos da cifose torácica na posição ortostática com as diferentes inclinações para trás do tronco (30, 50 e 70\% do peso corporal) usando o trapézio cadeirinha, simulando a condição de velejo. 0 objetivo secundário foi verificar as lesões sofridas e a presença de dor nas costas em decorrência da prática esportiva. A amostra foi composta por 11 praticantes de kitesurfing. Os ângulos da cifose torácica foram verificados na posição ortostática e com inclinações do tronco para trás de 30, 50 e 70\% do peso corporal com auxílio de duas câmeras de vídeo, simulando condições de velejo; lesões e dor nas costas foram verificadas por meio de uma anamnese. 0 ângulo da cifose torácica foi maior nas posições com inclinações do que na posição ortostática, predominando uma postura com hipercifose nas inclinações. A entorse foi a lesão mais frequente, sendo que o membro inferior foi o local mais acometido. Ainda, alguns participantes relataram dor nas costas durante e/ou logo após a prática esportiva. Assim, 0 aumento da cifose torácica nas simulações de velejo e a ocorrência de dor nas costas durante e/ou logo após a prática esportiva indicam a necessidade de um trabalho físico e/ou postural complementar.

Palavras-Chave: Biomecânica; Esporte; Saúde; Postura.

\section{Introdução}

O kitesurfing é um esporte radical relativamente novo que vem crescendo rapidamente em popularidade em vários países do mundo ${ }^{1}$, incluindo o Brasil ${ }^{2}$. Esse sucesso pode ser justificado pela diversidade de locais em que pode ser praticado e pelo reconhecimento que os atletas brasileiros 
têm no cenário mundial, despertando o interesse dos jovens ${ }^{2}$. Apesar desse rápido crescimento, as pesquisas relacionadas a essa modalidade ainda são escassas ${ }^{1}$.

Nesse esporte, o praticante, com uma prancha fixada em seus pés, gera propulsão sobre a água utilizando um kite (pipa) ${ }^{3}$, assim, utiliza a energia do vento para deslizar sobre a água ${ }^{4,5}$. Para controlar o kite, o praticante manipula uma barra de controle com suas mãos. Esta barra está interligada com quatro linhas, sendo que duas linhas saem da borda de fuga (perfil posterior) do kite e terminam nas extremidades da barra de controle, enquanto que as outras duas linhas saem da borda de ataque (perfil frontal) do kite, interligam-se passando por dentro da barra de controle e terminam em um dispositivo denominado chicken loop, estrutura em forma de argola, que é preso ao gancho do trapézio ${ }^{6}$.

O trapézio é um equipamento que fica ao redor do indivíduo, sendo que existem dois modelos ${ }^{7}$ : (1) o trapézio convencional é um dispositivo de gancho que é preso ao redor da cintura do indivíduo e (2) o trapézio cadeirinha é um dispositivo de gancho que abrange a região pélvica, com alças que são fixadas na região inguinal, ao redor da articulação coxofemoral. $\mathrm{O}$ trapézio recebe a energia de traçáo do kite, puxando o praticante para frente e para o lado ${ }^{8}$. A força que traciona para frente gera uma tendência de giro sobre o indivíduo, e para contrapor essa tendência de giro, o praticante inclina-se para trás usando o peso do seu corpo, permitindo apenas o deslocamento para o lado ${ }^{8}$. Ainda, para realizar manobras, saltos, giros ou aterrissagens, o velejador deve alterar a posição do seu corpo, adotando diferentes posturas que são acompanhadas pela mudança de posição da sua coluna vertebral ${ }^{9}$.

Essas variaçôes da posição do corpo, incluindo a regiáo do tronco, podem desencadear dor ou algum desconforto 9 . Além disso, quando o velejador

\section{Métodos}

\section{Delineamento experimental}

Este estudo caracterizou-se como ex post facto do tipo comparativo, em que foram comparados os ângulos da cifose torácica entre diferentes posiçōes: em ortostase e simulando posturas adotadas no velejo em que o praticante se inclina para trás. realiza travessias de longas distâncias, mantem a postura por um tempo prolongado por meio de ações musculares isométricas ${ }^{5}$, adotando posições articulares incômodas, principalmente sobre a coluna vertebral, podendo gerar desconforto e dor ${ }^{9}$ ou até lesão ${ }^{10}$. Como consequência, esses problemas podem levar o indivíduo a afastar-se da prática esportiva. Para evitar isso, é necessário, em um primeiro momento, identificar os fatores que podem potencializar o aparecimento ou o agravamento de desconfortos, dores e lesão ${ }^{11}$. Entre estes fatores, pode ser citada a postura adotada durante a prática esportiva ${ }^{9,11,12}$.

Desse modo, torna-se importante conhecer o comportamento da coluna vertebral dos praticantes do kitesurfing, o que pode potencializar dor nas costas e a ocorrência de lesóes. Assim, a análise da cifose torácica em posições que simulam o gesto esportivo do kitesurfing pode proporcionar um melhor entendimento de como se comporta essa parte da coluna durante a prática do esporte, auxiliando no diagnóstico e tratamento de lesóes ocasionadas pelo esporte e na atuação dos profissionais que trabalham com o treinamento ou com a reabilitação de atletas e praticantes amadores de kitesurfing.

A partir disso, o objetivo principal do presente trabalho foi comparar os ângulos da cifose torácica entre a posição ortostática e em diferentes inclinações para trás do tronco usando o trapézio cadeirinha, simulando as condições de velejo. Para complementar os resultados do estudo, o objetivo secundário foi verificar as lesóes sofridas e a ocorrência de dor nas costas em decorrência da prática do kitesurfing. Com base no objetivo principal, devido à postura que o praticante adota, inclinando seu corpo para trás para contrapor a tração do kite $e^{8}$ com flexão dos joelhos entre 30 e $45^{\circ}$ e com os cotovelos flexionados em torno de $90^{\circ 5}$, foi esperado encontrar um maior ângulo de cifose torácica nas inclinaçôes avaliadas em relação à posição ortostática.

\section{Amostra}

A amostra foi composta por 11 praticantes de kitesurfing do sexo masculino (TABELA 1), todos pertencentes à mesma escola de kitesurfing, os quais aceitaram em participar do estudo após uma breve apresentação do projeto por um dos pesquisadores. 
Os critérios de inclusão da amostra foram o tempo de prática superior a seis meses e saber orçar (velejar no sentido contrário ao vento). Já os critérios de exclusão compreenderam cirurgia na região do quadril e/ou coluna vertebral, doenças sistêmicas ou neurológicas e dor na regiáo dorsal do tronco no decorrer da coleta. Cada indivíduo assinou previamente um termo de consentimento livre e esclarecido e o presente estudo foi aprovado pelo comitê de ética da universidade em que foi realizado.

TABELA 1 - Perfil da amostra $(n=11)$.

\begin{tabular}{lc}
\hline & Média \pm desvio-padráo \\
\hline Idade $(\operatorname{anos})$ & $31,4 \pm 6,0$ \\
\hline Massa $(\mathrm{kg})$ & $73,8 \pm 8,9$ \\
\hline Estatura $(\mathrm{m})$ & $1,75 \pm 0,06$ \\
\hline
\end{tabular}

\section{Aquisiçáo dos dados}

Primeiramente, cada participante respondeu a uma anamnese sobre tempo de prática no esporte, lesões sofridas e dor nas costas em decorrência da prática de kitesurfing. Para reproduzir a prática esportiva, foi criado um ambiente que simulava a situação de velejo (FIGURA 1). Para isso, uma extremidade das quatro linhas de um kite foi presa em uma parede em uma altura de $3 \mathrm{~m}$ em relação ao solo; já a outra extremidade foi presa na barra de controle usada no esporte. Ainda, com o intuito de adotar um padrão, foi usada uma prancha fixada a um suporte de madeira, preso ao solo, com um ângulo de $30^{\circ}$ em relação à horizontal. A altura das linhas na parede buscou simular a inclinação do kite em relação ao horizonte do velejador, e a posiçáo angular da prancha foi escolhida com o intuito de simular a posiçáo da mesma em contato com a água. $\mathrm{O}$ trapézio utilizado foi o tipo cadeirinha, uma vez que o trapézio convencional impossibilitava a visualização dos marcadores.

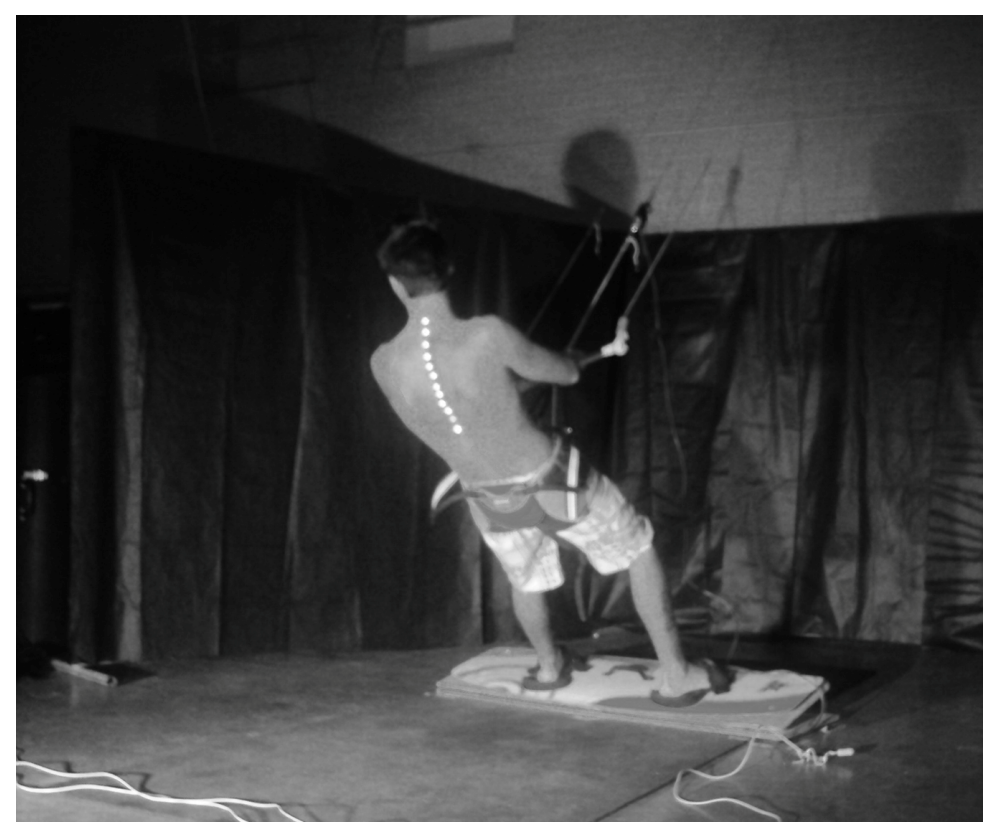

FIGURA 1 - Ambiente da coleta desenvolvido para simular a situação de velejo.

A fim de reproduzir o gesto esportivo em que o indivíduo se inclina para trás contra a tração do vento sobre o kite (FIGURA 2), considerando as diferenças antropométricas entre os participantes, optou-se por padronizar as inclinações para trás com cargas de 30, 50 e 70\% do peso corporal de cada participante. As inclinaçóes de 30, 50 e 70\% foram adotadas com a intenção de reproduzir posiçóes 
similares, respectivamente, (1) a de través (velejo perpendicular à direção do vento), (2) a realização da orça (velejo contrário ao sentido do vento) e
(3) quando o velejador necessita utilizar grande parte do seu peso corporal para contrapor uma grande força de tração exercida pelo kite.

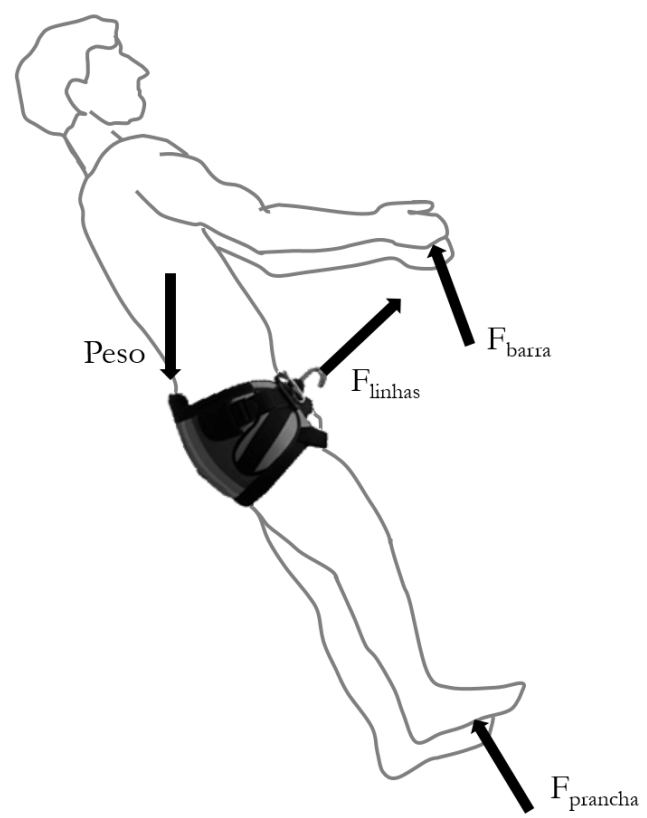

FIGURA 2 - Diagrama de corpo livre mostrando as forças atuantes no corpo do participante. $\mathrm{F}_{\text {barra }}$ é a força gerada pelo contato com a barra de controle, $\mathrm{F}_{\text {linha }}$ é a força gerada pelo contato com as linhas do kite, $\mathrm{F}_{\text {prancha }}$ é força gerada pelo contato com a prancha. $\mathrm{F}_{\text {barra }}$ e $\mathrm{F}_{\text {prancha }}$ foram somente representadas no lado direito do corpo.

A medição desses percentuais do peso corporal nas diferentes inclinaçóes foi realizada por meio da técnica de dinamometria. Desse modo, foi utilizada uma célula de carga (modelo ZX 250 Alfa Instrumentos - SP - com sensibilidade de $2 \mathrm{mV} / \mathrm{V}$; capacidade para $2500 \mathrm{~N} ; 2000 \mathrm{~Hz}$ ), previamente calibrada, fixada no dispositivo de linhas da barra de controle. Essa célula de carga foi ligada a um condicionador de sinais (Miotec Equipamentos Biomédicos Ltda), conectado a um computador (PCG-7154L Sony Vaio) com o software Miograph (Miotec equipamentos Biomédicos Ltda) que permitiu registrar e visualizar os valores de força.

Para verificar a posição angular da coluna torácica, marcadores reflexivos foram posicionados sobre os processos espinhosos das vértebras da coluna torácica em cada posição avaliada (FIGURA 1), ou seja, os marcadores foram posicionados em ortostase e nas diferentes inclinaçôes simuladas. Para capturar a imagem dos marcadores reflexivos, duas câmeras de vídeo digital (VPC-WH1 Sanyo; $30 \mathrm{~Hz} ; 640 x 480$ pixels) foram posicionadas a uma distância de $4 \mathrm{~m}$ do centro do ambiente de coleta com uma distância de $3 \mathrm{~m}$ uma da outra. Também foi usado um calibrador (Peak Performance modelo 5.3) - para permitir a posterior reconstrução tridimensional - o qual foi posicionado de tal forma que seus eixos $\mathrm{X}, \mathrm{Y}$ e $\mathrm{Z}$ encontrassem alinhados, respectivamente, com os planos frontal, longitudinal e sagital do participante.

Para cada indivíduo, foi sorteada a ordem das posições investigadas (ortostase e as inclinações para trás equivalentes a 30, 50 e 70\% do peso corporal). Conhecendo o peso de cada um, foram estimados os valores de força respectivos a cada inclinação. Cada participante foi solicitado a inclinar-se para trás até atingir o percentual almejado do peso corporal exibido no software Miograph, sendo que foi permitida uma oscilaçáo da força de $\pm 2 \%$. Atingindo o percentual do peso almejado, foram capturadas as imagens dos marcadores reflexivos durante 5 segundos.

Com exceção na avaliação da ortostase (em que somente foram capturadas as imagens dos marcadores reflexivos), nas outras posiçóes, também foram 
coletados os dados de força. Assim, como a coleta envolveu a aquisição de dados cinéticos e cinemáticos de forma independente, foi necessário utilizar um sistema eletrônico para alinhar os dados, o qual quando acionado forneceu, simultaneamente, um sinal elétrico - observado no software Miograph e um sinal luminoso - observado nas câmeras - possibilitando o alinhamento entre esses dois sistemas de medida. $\mathrm{O}$ sinal luminoso também foi usado para sincronizar as câmeras.

\section{Análise dos dados}

Primeiramente, foi confirmado o percentual do peso para as diferentes inclinaçóes em cada indivíduo no software Miograph, o que permitiu a análise dos dados das posiçóes. Após, os marcadores reflexivos foram digitalizados no software Digital
Video for Windows ${ }^{13}$ durante o segundo central coletado entre os $5 \mathrm{~s}$, e as coordenadas tridimensionais desses marcadores foram obtidas por meio do método de transformação linear direta no mesmo software. Todos os outros procedimentos para análise dos dados foram realizados no software Matlab (versão 7.5).

Para o cálculo do ângulo da cifose torácica, foram determinadas: uma reta $(h)$ da segunda vértebra torácica até a última vértebra torácica, dividida em duas partes (h1 e h2) e outra reta (f) perpendicular à h a partir do processo espinhoso mais proeminente (FIGURA 3). O ângulo da cifose torácica foi igual à soma dos ângulos $\phi_{1}$ e $\phi_{2}$, os quais foram estimados por meio das seguintes equaçóes ${ }^{14}$ :

$$
\begin{array}{ll}
\phi_{1}=180^{\circ}-2 \times \arctan (h 1 / f) & \text { EQUAÇÃO } 1 \\
\phi_{2}=180^{\circ}-2 \times \arctan (h 2 / f) & \text { EQUAÇÃO } 2
\end{array}
$$

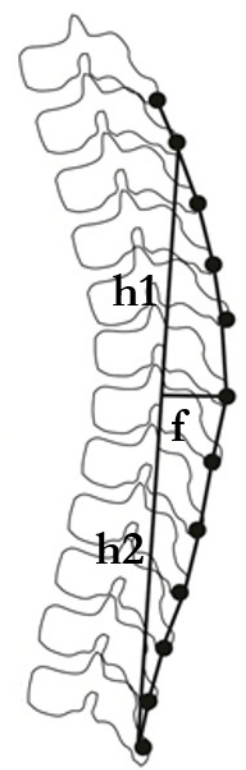

FIGURA 3 - Marcadores reflexivos sobre os processos espinhosos das vértebras torácicas (de T1 a T12) e as retas $\mathrm{feh}(\mathrm{h} 1 \mathrm{e}$ h2).

Após encontrar os ângulos da cifose torácica em cada inclinação avaliada, foi estimada a média de cada posição durante o segundo central para cada participante. Esse valor foi utilizado para a análise dos dados. Além disso, foi determinada a acurácia da coleta dos dados de posição ${ }^{15}$, a qual foi igual a $0,94 \mathrm{~mm}$. Em relação à dinamometria, pesos conhecidos foram aplicados sob a forma de tração na célula de carga. Os valores medidos e os reais foram plotados, e a linearidade da curva foi estabelecida por meio do coeficiente de determinação, o qual foi igual a 0,9996 .

\section{Análise estatística}

Primeiramente, foi confirmada a normalidade (teste de Shapiro-Wilk) e a esfericidade (teste de Mauchly) dos dados. Após, foi conduzida uma Análise de Variância para medidas repetidas com post hoc de Bonferroni para identificar as diferenças dos 
ângulos da cifose torácica entre as posiçóes avaliadas. O nível de significância adotado foi de $5 \%$, e os testes estatísticos foram realizados no software SPSS (versão
17.0 for Windows). Os procedimentos estatísticos e a forma de reportar os resultados foram estabelecidos conforme FIELD ${ }^{16}$.

\section{Resultados}

A TABELA 2 ilustra alguns resultados obtidos com a anamnese. Entre os 11 participantes, somente três relataram que nunca tiveram alguma lesão associada à prática do esporte, enquanto que dois relataram lesóes nos membros superiores (tendinite e entorse) e oito reataram lesóes nos membros inferiores como entorse (seis participantes), ruptura parcial de ligamento (dois participantes) e fratura (um participante).

TABELA 2 - Experiência dos participantes com kitesurfing $(\mathrm{n}=11)$.

\begin{tabular}{lc}
\hline & Média \pm desvio-padráo \\
\hline Frequência de velejo (dias/semana) & $2,6 \pm 1,4$ \\
\hline Tempo de prática (meses) & $56,2 \pm 40,5$ \\
\hline
\end{tabular}

Em relação à dor nas costas, um indivíduo reportou sentir dor na coluna lombar de forma crônica, enquanto que um reportou dor na coluna lombar e outro reportou dor na coluna torácica e lombar somente durante a prática do kitesurfing. Em relação à presença de dor nas costas logo após a prática do esporte, três participantes destacaram que sentem, às vezes, dor na coluna lombar, um destacou que, às vezes, sente dor na coluna cervical e outro destacou que sempre sente dor na coluna torácica e lombar.

A TABELA 3 ilustra os ângulos da cifose torácica nas diferentes posições avaliadas. Foi encontrado um efeito principal significativo da postura sobre o ângulo, $F(3)=7,849 ; p<0,001 ; \eta^{2}=0,416$, sendo que o post hoc revelou que o ângulo da cifose torácica foi significativamente menor na posição ortostática do que nas outras posiçôes.

TABELA 3 - Ângulo da cifose torácica (graus) para cada posição investigada, sendo que o valor-p é referente às diferenças encontradas com a posição ortostática ( $\mathrm{n}=11)$.

\begin{tabular}{lccc}
\hline & Média \pm desvio-padrão & Intervalo de confiança de 95\% & Valor- $\boldsymbol{p}$ \\
\hline Posição ortostática & $52,5 \pm 11,7$ & $45,0-59,9$ & \\
\hline $30 \%$ do peso corporal & $64,0 \pm 12,8$ & $55,9-72,2$ & 0,023 \\
\hline $50 \%$ do peso corporal & $64,0 \pm 10,8$ & $57,2-70,9$ & 0,031 \\
\hline $70 \%$ do peso corporal & $68,7 \pm 18,0$ & $57,3-80,2$ & 0,007 \\
\hline
\end{tabular}

\section{Discussão}

O kitesurfing pode ser considerado como um esporte de alto risco devido ao elevado número de acidentes e de lesôes decorrentes da sua prática ${ }^{4,17,18}$. Consequentemente, pesquisas que busquem entender as características ou as demandas do esporte e tornar a prática mais segura são fundamentais. Com esse intuito, alguns trabalhos investigaram: (1) aspectos mecânicos ou fisiológicos associados ao kitesurfing ${ }^{1,5,8,12,19}$; (2) as lesôes ou acidentes ocasionados por essa prática esportiva ${ }^{4,11,17,20}$ e (3) a segurança atrelada ao uso de equipamentos ${ }^{3,7}$. O presente estudo enquadra- 
se na primeira abordagem, mas também apresenta resultados referentes à segunda.

A partir da importância da posição da coluna nas posturas adotadas durante a prática do kitesurfing ${ }^{9,11,12}$, este estudo analisou o comportamento da coluna torácica simulando a condição de velejo com o trapézio cadeirinha em que o praticante realiza inclinaçóes do tronco para trás para contrapor a força de traçáo do vento sobre o kite usando uma parte do seu peso corporal ${ }^{8}$. Além disso, também foi aplicada uma anamnese sobre ocorrência de lesáo e dor nas costas decorrentes da prática desse esporte.

De forma similar ao trabalho de BERNEIRA et al. ${ }^{11}$, os participantes apresentaram a entorse como a lesão mais frequente e os membros inferiores como o local mais acometido. Também foi constatado que alguns participantes apresentam dor nas costas durante e/ ou logo após a prática do kitesurfing, o que está de acordo com os achados de Lundgren et al. ${ }^{9}$ Essa dor pode ser devido à postura adotada no velejo por meio de açôes musculares isométricas ${ }^{5}$ e/ou devido a um baixo condicionamento físico ${ }^{18}$.

A análise do ângulo da cifose torácica revela que a cifose aumenta da posição ortostática para as posiçôes com inclinações (TABELA 2), o que está de acordo com a hipótese formulada. Conforme Leroux et al. ${ }^{14}$, a partir do método empregado para estimar esse ângulo, pode-se considerar como uma faixa normal valores entre 21 e $52^{\circ}$, em que resultados superiores a estes são caracterizados como uma hipercifose. Por meio da análise dos resultados de cada um dos 11 participantes, a cifose torácica caracterizou-se como normal para cinco sujeitos na posição ortostática, para dois na inclinação de $30 \%$ e para um nas inclinaçóes de 50 e $70 \%$ do peso corporal, ou seja, para todas as posições com inclinaçóes, predomina uma postura com hipercifose. É interessante destacar que a única pessoa que apresentou uma cifose torácica normal em todas as posiçóes avaliadas também foi um dos poucos participantes que não relatou sentir dor nas costas decorrente da prática esportiva.

Como o ambiente de coleta buscou simular a postura adotada durante o velejo (FIGURA 1), o aumento do ângulo da posiçáo ortostática para as posiçôes com inclinação do corpo pode ser justificado pela postura adotada pelo praticante, o qual mantém os joelhos com uma flexão entre 30 e $45^{\circ}$ aproximadamente, ao mesmo tempo em que mantém o cotovelo flexionado em torno de $90^{\circ 5} \mathrm{e}$ inclina seu corpo para trás para contrapor a tração do $k i t e^{8}$. Isto é, a postura adotada pelo corpo favoreceu o aumento da cifose torácica, o que pode ser devido à fraqueza muscular, especialmente dos eretores da coluna $^{21,22}$. Desse modo, o presente estudo indica a necessidade de promoção de exercícios físicos voltados para o fortalecimento dos estabilizadores do tronco para a prática de kitesurfing ser mais segura. Interessantemente, BERNEIRA et al. ${ }^{11}$, quando questionaram 50 praticantes de kitesurfing se a prática esportiva exigia uma excelente aptidão física, $60 \%$ responderam que não e $80 \%$ relataram já ter um nível de condicionamento físico adequado para a prática.

Além da posição adotada nas inclinaçóes favorecer o aumento da cifose torácica, seis participantes já apresentavam uma hipercifose na posição ortostática. Esse ângulo exacerbado nessa posição pode ser resultado da manutenção prolongada de uma postura em hipercifose na prática esportiva, assim como pode ser devido à postura adotada em atividades de vida diária ${ }^{22}$. Por isso, pode ser destacada também a importância de se realizar um trabalho de educação postural.

Apesar de que a lordose lombar náo pôde ser investigada em funçáo de que os marcadores reflexivos ficariam encobertos pelo trapézio cadeirinha, Bourgors et al. ${ }^{18}$ também destacam a importância do fortalecimento dos músculos abdominais com o intuito de contrapor a tendência de uma hiperlordose lombar quando se pratica kitesurfing. Isto é, Bourgois et al. ${ }^{18}$ destacam a necessidade de um melhor treinamento físico combinado com exercícios de propriocepção, de estabilidade do core e de mobilização articular com o intuito de evitar lesóes. De fato, a manutenção prolongada de uma postura, como durante uma regata ou uma travessia, pode levar a uma fadiga muscular, diminuindo a capacidade do músculo de absorver as cargas aplicadas sobre o corpo, sobrecarregando estruturas como ligamentos, cápsula articular e o anel fibroso dos discos intervertebrais, o que aumenta a suscetibilidade para a ocorrência de lesóes ${ }^{10}$.

Como a coleta de dados em uma situação esportiva real é um muito difícil de ser realizada, devido ao meio em que o kitesurfing é praticado e aos procedimentos metodológicos necessários, o presente estudo simulou as condiçóes de velejo. Por outro lado, foi intençâoo analisar posturas mais próximas da situação real, indo além da avaliaçáo da posição ortostática. Para complementar os resultados do presente trabalho e superar as limitações desta pesquisa, sugere-se: (1) a avaliação de toda a coluna vertebral nos planos sagital e frontal em conjunto com a pelve durante a simulaçáo do velejo com o trapézio cadeirinha; (2) a análise de 
toda a coluna com o trapézio convencional, o que possibilitaria a comparação entre os tipos de trapézio e, mais importante ainda, (3) a análise da coluna vertebral durante a condição real de velejo. Somado a isso, seria muito interessante verificar a associação entre a aptidão física e a postura, assim como o efeito de um treinamento físico sobre a postura adotada dentro e fora da prática esportiva. Essas sugestóes não são fáceis de serem executadas, mas contribuiriam muito na compreensão sobre as demandas associadas à prática do kitesurfing.

Portanto, em relação a lesões, a entorse foi a mais frequente, sendo que os membros inferiores foram o local mais acometido. Também foi constatado que alguns participantes apresentam dor nas costas durante e/ou logo após a prática do kitesurfing. Em relação à postura avaliada, o ângulo da coluna torácica foi maior em posiçóes que simulam o velejo do que na posição ortostática, predominando uma postura com hipercifose. O conhecimento desses resultados pode ser uma ferramenta útil para profissionais da área da saúde que atuam com atletas ou com praticantes de kitesurfing, uma vez que pode ajudar na elaboração de propostas para reduzir os riscos de lesões no esporte, atuando diretamente na postura do velejador, de forma a reeducar e a fortalecer os músculos que estabilizam a região do tronco. Em caso de lesóes, pode auxiliar na elaboração de planos de reabilitação mais efetivos, principalmente, em um trabalho postural e funcional para a regiáo do tronco.

\title{
Agradecimentos
}

Os autores agradecem a Victor Wigner Tremea pelo auxílio prestado no desenvolvimento do projeto deste estudo e ao CNPq pelo apoio financeiro.

\begin{abstract}
Kyphosis thoracic angle in different positions that simulate the sailing in kitesurfing

In kitesurfing, the sailor uses the energy of the wind to propel himself with a board on the water with the aid of a kite, which can be attached to the athlete through a harness. In order to perform several movements, the sailor changes the position of the body, modifying the position of his/her spine. However, little attention has been paid to the posture that is adopted during kitesurfing, which may contribute to back pain. Thus the main purpose of the present study was to compare the kyphosis thoracic angle in the orthostatic position with different inclinations of the trunk backward (30,50 e 70\% of the body weight) using a seat harness, simulating the sailing conditions. The secondary purpose was to verify the occurrence of injuries and back pain from sports practice. The sample was composed of 11 kitesurfers. The kyphosis thoracic angles were verified in the orthostatic position and in different inclinations bending the trunk backward with 30, $50 \mathrm{e}$ $70 \%$ of the body weight with two video cameras, simulating sail conditions; injuries and back pain were evaluated by an anamnesis. The kyphosis thoracic angle was higher in the conditions with inclination of trunk backward than in the orthostatic position, and a hyperkyphosis posture prevailed in the conditions with inclination of trunk backward. Sprains were the most common injury, and the lower limb was the place more affected. Moreover, some participants related back pain during and/or sooner the sports practice. Therefore, the increase in the kyphosis angle and the occurrence back pain during and/or sooner the sports practice indicate the need of performing physical and/or postural exercises.
\end{abstract}

KEYwords: Biomechanics; Sport; Health; Posture. 


\section{Referências}

1. Nebas T, Heller B. A smartphone-based system for kite and board measurements in kitesurfing. Procedia Eng. 2014;72:477-8

2. Bitencourt V, Navarro P. Kitesurfe. In: DaCosta, L, organizador. Atlas do esporte no Brasil: atlas do esporte, educação física e atividades físicas de saúde e lazer no Brasil. Rio de Janeiro: Shape; 2005. p. 431-2.

3. Lundgren L, Bligård LO, Brorsson S, Osvalder A. Implementation of usability analysis to detect problems in the management of kitesurfing equipment. Procedia Eng. 2011;13:525-30.

4. Nickel C, Zernial O, Musahl V, Hansen U, Zantop T, Petersen W. A prospective study of kitesurfing injuries. Am J Sports Med. 2004;32(4):921-7.

5. Vercruyssen F, Blin N, L'Huillier D, Brisswalter J. Assessment of physiological demand in kitesurfing. Eur J Appl Physiol. 2009;105(1):103-9.

6. Blouin Junior M, Isabella B, Rodden J. Wind power from kites [monografia]. Worcester: Faculty of the Worcester Polytechnic Institute; 2007.

7. Lundgren L, Brorsson S, Osvalder AL. Comfort aspects important for the performance and safety of kitesurfing. Work. 2012;41(supl 1):1221-5.

8. Tremea VW, Gomes LE, Loss JF. Determinação da força das linhas do kite sobre um praticante em terra, utilizando o trapézio e a cadeirinha do kitesurfing. Rev Bras Ci Mov. 2010;18(1):100-4.

9. Lundgren L, Brorsson S, Hilliges M, Osvalder A. Sport performance and perceived musculoskeletal stress, pain and discomfort in kitesurfing. Int J Perform Anal Sport. 2011;11(1):142-58.

10. Ruschel C, Jacomel GF, Schütz GR, Haupenthal A, Hubert M, Roesler H. Análise cinesiológica do movimento de escora de velejadores da classe laser em diferentes posiçóes do barco em relação ao vento. Rev Educ Fis/UEM. 2008;19(4):501-8.

11. Berneira JO, Domingues MR, Medeiros MA, Vaghetti CA. Incidência e características das lesóes em praticantes de kitesurf. Rev Bras Cineantropom Desempenho Hum. 2011;13(3):195-201.

12. Tarabini M, Saggin B, Scaccabarozzi D. Whole-body vibration exposure in sport: four relevant cases. Ergonomics. 2015;58(7):1143-50.

13. Figueroa PJ, Leite NJ, Barros RML. A flexible software for tracking of markers used in human motion analysis. Comput Methods Programs Biomed. 2003;72(2):155-65.

14. Leroux MA, Zabjek K, Simard G, Badeaux J, Coillard C, Rivard CH. A noninvasive anthropometric technique for measuring kyphosis and lordosis. Spine. 2000;25(13):1689-94.

15. Barros RML, Russomanno TG, Brenzikofer R, Figueroa PJ. A method to synchronise video cameras using the audio band. J Biomech. 2006;39(4):776-80.

16. Field A. Descobrindo a estatística usando o SPSS. Porto Alegre: Artmed; 2009.

17. Spanjersberg WR, Schipper IB. Kitesurfing: when fun turns to trauma: the dangers of a new extreme sport. J Trauma. 2007;63(3):76-80.

18. Bourgois JG, Boone J, Callewaert M, Tipton MJ, Tallir IB. Biomechanical and physiological demands of kitesurfing and epidemiology of injury among kitesurfers. Sports Med. 2014;44(1):55-65.

19. Tremea VW, Gomes LE, Loss JF. Descrição do comportamento da força do kite em relação ao seu posicionamento e ao deslocamento da barra de controle. Rev Bras Ciênc Esporte. 2011;33(3):715-32.

20. Exadaktylos AK, Sclabas GM, Blake I, Swemmer K, McCormick G, Erasmus P. The kick with the kite: an analysis of kite surfing related off shore rescue missions in Cape Town, South Africa. Br J Sports Med. 2005;39(5):[4 p.].

21. Katzman WB, Wanek L, Shepherd JA, Sellmeyer DE. Age-related hyperkyphosis: its causes, consequences and management. J Orthop Sports Phys Ther. 2010;40(6):352-60.

22. López-Miñarro PA, Muyor JM, Alacid F. Sagittal and pelvic postures of highly-trained young canoeists. J Hum Kinet. 2011;29:41-8.

ENDEREÇO

Rodrigo Fagundes Angellos

Rua José Domingos Varella, 280/305 - Cavalhada 91910-420 - Porto Alegre - RS - BRASIL e-mail: rfangellos@hotmail.com; rodrigo@ grupofuncionalrs.com.br
Recebido para publicação: 29/03/2016

$1^{\text {a }}$ Revisão: 31/05/2016

$2^{\text {a }}$ Revisão: 16/08/2016

Aceito: 29/08/2016 